ISSN 09753303

MUS, Vol.1, No. 2, Dec.-2002 - April 2003 pp. 57.68

https:/ / doi.org/ 10.12725//mjs.2.6

\title{
Immunization of Children
}

\section{Y. Udya Bhaskara Rao*}

\section{Introduction}

Immunization has been one of the great successes of twentieth century medicine for prevention and control of human and animal infectious diseases. The routine immunization programs against childhood diseases have proved to be very successful in both developed and developing countries. The Government of India initiated the Expanded Program on Immunization (EPI) in the year 1978 with the object of providing immunization for all the children against six vaccine preventable diseases i.e. Diphtheria, Tetanus, Pertussis, Tuberculosis, Polio and Measles. The achievements are remarkable and the incidences of these six diseases have been brought down significantly (MHFW, 2000). Tetanus toxoid is administered to the mother either during her pregnancy or prior to pregnancy during the childbearing years to protect new borne against neonatal tetanus. The global Polio eradication initiative began in 1988 (World health Assembly, 1988) through 2001, the number of reported Polio cases in the world has been reduced by more than 99 percent from an estimated $3,50,000$ to $<1000$ cases and number of countries where Polio is endemic decreased from 125 to 10 (MMWR, 2002). The decrease of the other diseases is remarkable as per the records maintained by centers for Disease. Control and Prevention (CDC) and it shows over 99 percent decrease in the case of Diphtheria, Measles, Mumps and rubella and over 97 percent in the case of Whooping cough (MMWR, 1998). As stated by Plotkin and Plotkin, "the impact of vaccination on the health of the world's peoples is hard to exaggerate with the exception of safe water, no other modality, not even antibiotics, has had such a major effect on mortality reduction and population growth" (Plotkin and Plotkin, 1999).

* Dr. Y. Udaya Bhaskara Rao, Deputy Director, Pasteur Institute Of India, Coonoor - 643 103, Nilgiris. 
The breakthroughs in molecular biology, biochemistry, immunology and related fields have resulted in the development of many new vaccines like Hepatitis ' $B$ ' as well as in the improvement of several existing ones. The concept of combination of vaccines if developed successfully could help to reduce in the number of injections during the first two years of life. Presently immunization protects the children against Hepatitis B, Measles, Mumps, Rubella (German Measles), Polio, Diphtheria, Tetanus, Pertussis, Haemophilus, Influenza type ' $b$ ' and chicken pox. All these immunization need to be given before the children are two years old.

\section{History of Immunization}

One of the most important activities in total comprehensive care for children is successful immunization practice. The earliest documented attempts at immunization aimed at preventing small box were reported nearly 1000 years ago. The first milestone in immunization was laid by Edward Jenner in 1796 with a systematic, successful attempt at eradication of small box by vaccination. He actively immunized an eight - year old boy with the fluid from the pustule of a cowpox lesion on the hand of a milkmaid and challenged with small poxvirus. The boy was protected against smallpox virus. The boy was protected against small pox despite exposure to virus and this weakened form of 'Small Pox' was called a vaccine from vacca, the Latin word for cow. During 19th century, the small pox vaccination became very popular and small pox was the first communicable disease to be eradicated globally in the year 1976.

Pasteur in 1885 developed first rabies vaccine successfully proving attenuated germs when injected into the body confers immunity against the disease. Pasteur suggested that all inoculations be called "Vaccinations" to honor Jenner and today the more accurate word "Immunization" is preferred.

The introduction of inactivated vaccines in 1890 heralded a dramatic shift in the methodology used to produce vaccines. The prime reason was safety. The early twentieth century saw the introduction of numerous bacterial and viral vaccine preparations containing either whole-inactivated bacteria or virus or semi purified detoxitied bacterial toxins. Some of the important vaccines used in immunization are presented in chronological order in Table 1. 


\section{Table 1}

Chronology of important human vaccines used

Year of Introduction

1721

1798

1885

1896

1896

1897

1923

1926

1927

1955

1961

1963

1974

1976

1981

1986
Disease

Small pox

Small pox

Rabies

Typhoid fever

Cholera

Plague

Diphtheria

Pertussis

Tuberculosis

Polio

Polio

Measles

Japanese encephalitis

Rabies

Hepatitis B

Hepotitis B
Type of Vaccine

'Variolation' with live virus

'Naturally attenuated' cow pox virus

Attenuated live and inactivated virus

Inactivated intact bacteria

Inactivated intact bacteria

Inactivated intact bacteria

Partially puritied formalin toxoid

Inactivated intact bacteria

Live attenuated BCG strain

Inactivated virus

Live attenuated virus

Inactivated virus and live attenuated virus

Inactivated virus

Tissue Culture, inactivated virus

Inactivated plasma derived

Inactivated, Recombinant

\section{Types of Immunization}

Immunization involves the induction or administration of antibodies and other natural defense mechanisms to protect against specific pathogens. There are two types of immunization.

a) Active Immunization

b) Passive immunization 


\section{Active Immunization}

Active Immunization is achieved by the administration of modified pathogenic agent or a component pathogen, to stimulate the recipient's immune mechanism to produce long lasting protection without cousing the clinical manifestation of disease. To produce the active immunity, the preparations can be divided into three broad categories.

\section{a) Live attenuated vaccine:}

Live attenuated vaccine is a microorganism that can replicate on its own in the host or infect cells and function as an immunogen without causing its natural disease. Most of these vaccines confer life long immunity but there is a risk of reversion to virulence. The examples include widely used Sabin type of Polio vaccine (OPV) (Sabin \& Boulger, 1973), BCG vaccine against tuberculosis (Harboe et al, 1996) the vaccines against Measles, Mumps, Rubella (Gluck \& Just, 1991) and Varicella (Takahashi et al., 1974).

\section{b) Inactivated vaccine (Killed or Subunit Vaccine)}

Inactivated vaccine is an immunogen that cannot replicate in the host. The immunity conferred is of short duration and requires repeated boosters. The examples includes Salk Poliovirus vaccine (Salk, 1960) commonly called Inactivated Polio Virus Vaccine (IPV), whole cell vaccine against Pertussis and the Influenza vaccine.

\section{c) DNA-based vaccines}

DNA- based vaccine, which cannot replicate in humans, is taken up by cells in which it directs the synthesis of vaccine antigens.

\section{Passive Immunization}

Passive Immunization is obtained by transferring antibodies against a given disease from an immune person or animal to a non-immune individual by injection of serum (antisera) or some partially serum extract.

Diphtheria antitoxin was the first such product commercially available followed by Tetanus antitoxin which were of equine origin. Their use was associated with untoward side effects such as serum sickness. Presently, human origin sera or globulins are 
available but they are very expensive. Because of the cost, the equine globulins being continued to use in all the developing countries. A few immunoglobulins licensed for the preventive or treatment of infectious diseases are listed below:

\section{Immunization Schedule for Children}

The principle of any immunization program is, protection must be achieved prior to the time that infants are at high risk from a disease. Infants are highly susceptible for Pertussis soon after birth and it is recommended to start immunization with DPT at 6 weeks of age, with subsequent doses at 10 and 14 weeks.

Neonatal tetanus (NNT) is an important cause of infant mortality in many countries and immunization of women of childbearing age and especially pregnant women is recommended.

The simultaneous administration of several vaccines simplifies routine childhood immunization and reduces the number of contacts or visits. All the EPI vaccines can be administered simultaneously (Galazka, 1991) and it is a common practice to give DTP vaccine and oral Polio Vaccine (OPV) at the same time. BCG vaccine is compatible with OPV, DTP and Measles vaccine (Galazka, 1993). In United States to eliminate the risk of vaccine associated paralytic Polio, routine vaccination of children was recommended with IPV and presently OPV is unavailable for routine use (CDC, 2000). However; OPV remains the vaccine of choice as it induces intestinal immunity and prevents spread among close contacts.

In 1989, the Global Advisory Group (GAG) of EPI officially recommended that Hepatitis-B $(H B)$ vaccination to be administered to all infants as part of $E P I$ in countries where prevalence of chronic carriers of Hepatitis-B virus (HBV) exceed $2 \%$ and recommended all the countries to under take universal infant immunization with $\mathrm{HB}$ vaccine by 1997 (PATH, 1993). In 1992 world health assembly endorsed these targets and $\mathrm{HB}$ vaccine now to be considered the seventh EPI vaccine. The three-dose schedule for primary immunization against $\mathrm{HBV}$ should be begun in early infancy. The American Academy of Pediatrics (AAP) recommends giving the first dose before discharge from the hospital after delivery, the second dose should be administered at 12 months of age followed by a third dose at 6 to 18 months of age (AAP, 1992).

The schedules of childhood immunization vary slightly from country to country and the immunization schedules recommended by EPI (Galazka, 1993) and our country (NIM) are presented in the Table 3. \& 4. In addition to these, United States recommends the following vaccines for childhood immunization. 
Table 2

Immunoglobulins available for Treatment of infectious Diseases

\begin{tabular}{|l|l|}
\hline Viral Diseases & Bacterial Disease \\
\hline Rabies & Tetanus \\
\hline Measles & Diphtheria \\
\hline Chicken Pox & Pertussis \\
\hline Hepatitis B & \\
\hline
\end{tabular}

1. Haemophilus influenza type $b(\mathrm{Hib})$ conjugate Voccine: three doses are given at the age of 2,4 and 6 months. (The bacteria cause meningitis, pneumonio and throat infection).

2. Measles, Mumps and Rubella Vaccine (MMR): two doses are recommended and the 1 st dose to be given at the age of 12 months or after 12 months. Second dose is recommended at age 4-6 years. Niral infection is characterized by rashes, fever and potentially serious side effects such as heart damage and pneumonia).

3. Varicella Vaccine: recommends at or after 12 months of age for all susceptible children. (Protects against chicken pox).

4. Pneumococcal Vaccine: Recommended for all children aged 2-23 months.

5. Hepatitis-A Vaccine: Recommended in high-risk groups between $2-18$ years.

6. Influenza Vaccine: Recommended annually for children age of more than 6 months with certoin risk factors.

\section{Table 3}

The Immunization Schedule Recommended By The EPI

\begin{tabular}{|c|c|c|c|}
\hline \multirow[t]{2}{*}{ AGE } & \multirow[t]{2}{*}{ VACCINES } & \multicolumn{2}{|c|}{ HEPATITIS B (HB) VACCINE } \\
\hline & & Scheme A & Scheme B \\
\hline Birth & BCG, OPVO* & HBI & \\
\hline 6 weeks & DTPI, OPV1 & HB2 & $\mathrm{HBI}$ \\
\hline 10 weeks & DTP2, OPV2 & & HB2 \\
\hline
\end{tabular}




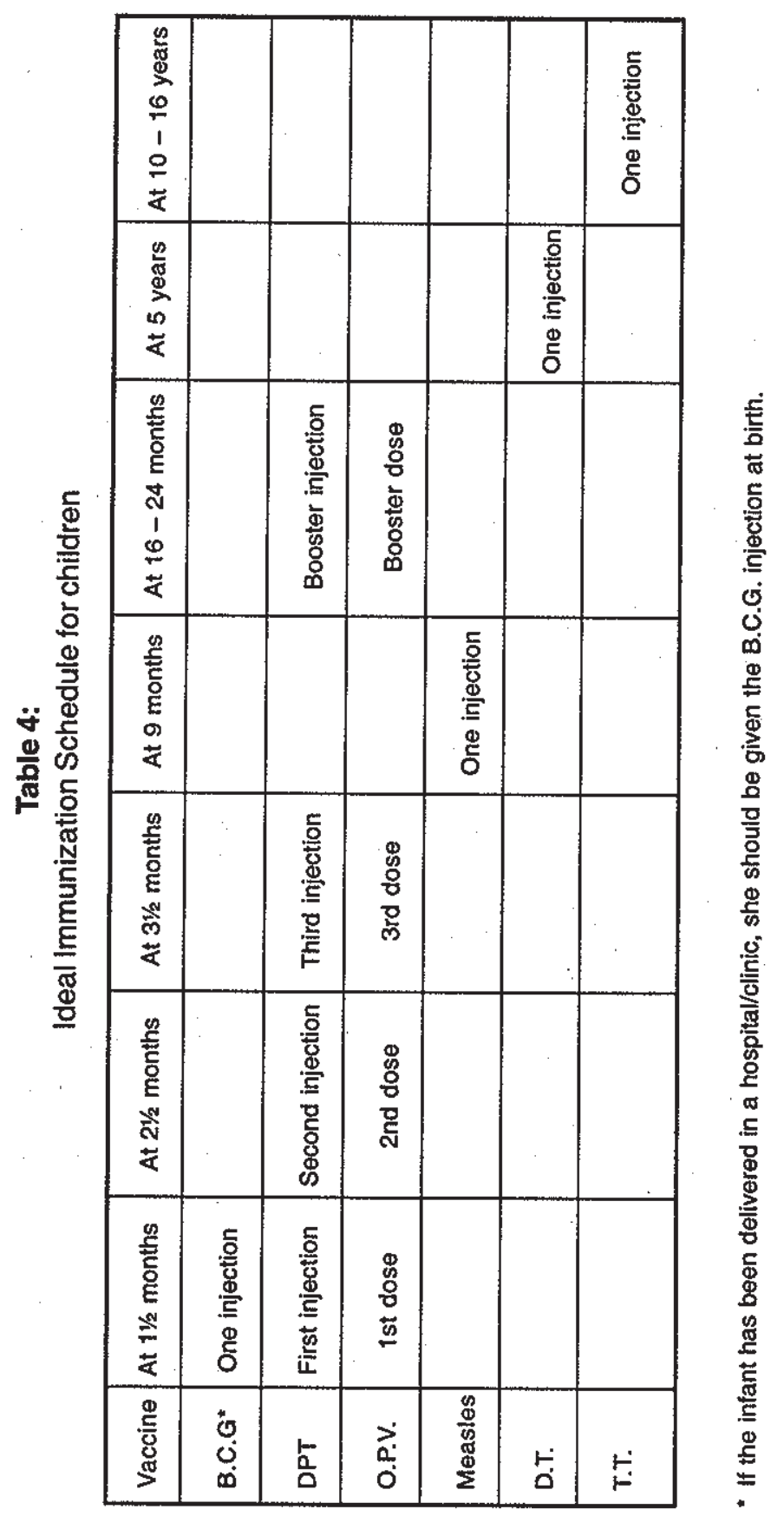




\section{Indications for Immunization}

1. The immunization schedule mentioned in table 4 till 9 months of age is the most important part of immunization.

2. Even if the mother is late in bringing the infant for the D.P.T. and O.P.V. injections/ doses, all the three injections/doses must still be given, keeping a difference of 1 month between them, before the infant is one year old.

3. The infant must be given the measles injection at 9 months of age. Even if the mother is late in bringing the infant, the infant must still be given the injection before she is 1 year old because without measles injection, the infant is not fully immunized.

4. Ensure that the infant is given 1 B.C.G. injection, 3 D.P.T. injections, 3 O.P.V. doses and 1 measles injection before she is 1 year old.

5. Children, especially girls, should be given one injection of T.T. at 10 years and one at 16 years of age.

TABLE 5

Yearwise And Antigenwise Achievement as

Percentage of Annual Target

\begin{tabular}{|l|l|l|l|l|l|}
\hline Year & DPT & OPV & BCG & MEASLES & TT (PW) \\
\hline $1995-96$ & 91.03 & 91.61 & 97.07 & 82.64 & 80.33 \\
\hline $1996-97$ & 89.32 & 90.75 & 97.10 & 82.01 & 78.70 \\
\hline $1997-98$ & 88.70 & 89.20 & 94.80 & 30.00 & 78.50 \\
\hline $1998-1999$ & 92.80 & 94.30 & 97.00 & 87.30 & 82.90 \\
\hline
\end{tabular}

\section{Precautions for Immunization}

1. The infant should be immunized even if she is suffering from diarrhoea, fever and malnutrition.

2. After the B.C.G. injection, a small blister will appear at the site of injection after 1 to $1 \mathrm{~A}$ months. This is normal and is indicative of the fact that the vaccine is working. 
3. Abscess should not form after DPT injection and if any abscess develops, the parents are advised to consult the doctor.

4. Immunization process should not be stopped unless the child is very sick and needs hospitalization.

\section{Safety of Vaccines}

Modern vaccines are safe and effective, however no biological or drug is completely safe and 100 percent effective. A few adverse events have been reported after administration of vaccines. With in the first 24 hours of vaccination, reactions include erythema and swelling at the site of injection, fever, prolonged crying, syncope, seizures and rarely hypo tonic, hypo responsive episodes or anaphylaxis

\section{TABLE 6}

Reported Incidence of Diseases

\begin{tabular}{|l|c|c|c|}
\hline Disease & 1987 & 1999 & $\%$ Decline \\
\hline Polio & 28,257 & 4,320 & 84.70 \\
\hline Diphtheria & 12,952 & 2,725 & 79.00 \\
\hline Pertussis & $1,63,786$ & 36,716 & 77.60 \\
\hline NNT & 11,849 & 4,488 & 62.10 \\
\hline Measles & $2,47,519$ & 38,950 & 84.30 \\
\hline
\end{tabular}

(Ada $G, 2001$ ). Late reactions, which occur within a few weeks after vaccination, include encephalitis and encephalopathy and some times to brain damage. But overall there is no clinical or scientific evidence that inoculation of any vaccine causes a specific allergy, asthma, autism, multiple sclerosis or sudden infant death syndrome (Ada G, 2001).

A few major reasons for low rates of immunization among children have been identified by the National Vaccine Advisony Committee (NVAC, 1991). First, many opportunities to vaccinate children are missed because of failure to administer recommended immunizations during health care visits, due to wrong information on contraindications or they are unwilling to give more than two vaccines in the same visit. In most cases, multiple vaccines can be given with out increased risk of adverse effects and impairment of immunological response (Eskola et al., 1987\& 
Deforest et al., 1988). Second reason is deficiencies in the health care delivery system in the public sector, which includes insufficient staff and polices that serve as barriers. The other reasons are inadequate access to medical care and lack of public awareness in some communities.

\section{Conclusions}

The first vaccine was developed 200 years back and millions of lives have been saved from infectious diseases. In the past 50 years the incidence of vaccine preventable diseases were reduced by nearly 90 percent from their peak levels. A better understanding of immune system and its process has driven the explosion of interest in vaccines by both private and public sectors. Presently we are probing into even greater depths into the mechanisms of pathogens use to infect us and cause disease. Genomic research has sequenced genes of many organisms and sequencing is currently in progress for more number of organisms and vaccine research is benefited to develop safe and effective vaccines. New generation technologies like DNA vaccines and edible vaccines emerged from the recent research are in progress to develop vaccines which are more effective longer periods in less frequent doses, quality vaccines at lower price, more stability in wide range of environmental conditions, easy to administer and finally safe and effective. Immunization remains on of the most powerful tools in control and prevention of infectious diseases.

\section{References}

1. AAP, 1992. Committee on Infectious diseases - Universal hepatitis -B immunization. Pediatrics, 89, pp. 795-800.

2. Ada G, 2001. Vaccines and vaccination. N Engl J Med, 345, pp. 1042-1053.

3. CDC, 2000. Poliomyelitis prevention in the United States: updated recommendation of the advisory committee on immunization Practices (ACPI). MMWR, 49, pp. 1.22.

4. CDC, 2002. Progress towards global eradication of poliomyelitis, 2001. MMWR, 51, pp. 253-256.

5. Deforest $A$ et al, 1988. Simultaneous administration of measles-mumps-rubella vaccine with booster doses of diphtheria-tetanus-pertussis and polio vaccines. Pediatrics, 81, pp. 237-246.

6. Eskola $\mathrm{J}$ et al, 1987. Efficacy of Haemophilus influenza type-b polysaccharide-diphtheria toxoid conjugate vaccine in infancy. N Engl J Med, 317, pp. 717-722.

7. Galazka A, 1991. Simultaneous administration of vaccines. Document EPI/RD/91/WP7/ APR/Rev. 1. Geneva: WHO. 
8. Galazka A, 1993. The immunological basis for immunization Series, Module-1 General immunology. WHO/EPI/Gen/93-11, 1-13.

9. Gluck $R$ \& Just $M, 1991$. Vaccination against measles, mumps and rubella in vaccines and Immunotherapy edited by Cnyz SJ Jr, Pergamon press, New York.

10. Harboe MP et al, 1996. Vaccines against tuberculosis. Vaccines, 14, pp. 701-716.

11. MHFW, 2000. Annual Report 1999-2000, Ministry of Health and family Welfare Govt. of India. pp. 6!-72.

12. NIM - National immunization Mission, Govt. of India- Immunization schedule, information brochure.

13. PATH, 1993. Global Perspectives on Hepatitis. June 1993, 4, pp. 1-8.

14. Plotkin SL \& Plotkin SA, 1999. A short history of vaccination in vaccines edited by Plotkin SA \& Orenstein WA, 3rd edition, WB Saunders company, Philadelphia, pp. 1-2.

15. Sabin $A B$ \& Boulger $L R, 1973$. History of Sabin attenuated Poliovirus live vaccine strains. Biol Stand, 1, 115-118.

16. Salk J, 1960. Persistence of immunity after administration of formalin treated Poliovirus vaccine. Lancet, 2, pp. 715-723.

17. Takahashi $M$ et al, 1974. Live attenuated Varicella Vaccine used to prevent the spread of Varicello in hospital. Lancet, 2, pp. 1288-1290.

18. World Health Assembly, 1988. Polio eradication by the year 2000. Resolution of 41 st world Health Assembly, Geneva, Swizerland: world health organization (resolution 41.28). 